\title{
Arbor
}

\section{La prospectiva, una herramienta usada por el Mando de Adiestramiento y Doctrina para vislumbrar el futuro del Ejército}

\section{Benito Vinuesa Guerrero}

Arbor CLXV, 651 (Marzo 2000), 475-488 pp.

El Mando de Adiestramiento y Doctrina (MADOC), está imprimiendo un nuevo rumbo en la configuración del Ejército del siglo XXI. Los procesos de profesionalización y de modernización quedarían incompletos sin una mejora en la formación, doctrina, orgánica y materiales y sin una visión prospectiva de la sociedad y del Campo de Batalla Futuro.

La prospectiva, frente a las técnicas de predicción clásicas, se está convirtiendo en la herramienta principal dentro del campo de la futurología. Se ha consolidado a escala internacional, consiguiendo la aceptación generalizada por parte de empresas y administraciones públicas. En este artículo se intenta hacer un breve resumen de los fundamentos y de los métodos de la prospectiva, de cómo han surgido, y de la evolución histórica desde el pensamiento «fatalista» de la antigüedad, al «determinista» de la ciencia del siglo pasado, para llegar al «indeterminista» de la prospectiva.

\section{La previsión del futuro}

Es inherente a la naturaleza humana, la inquietud por conocer el futuro. Desde la más remota antigüedad, el hombre ha usado los medios a su alcance para intentar desvelar las incertidumbres del porvenir. En los yacimientos arqueológicos, junto a los restos de huesos, 


\section{Benito Vinuesa Guerrero}

se encuentran a menudo astrágalos y otros utensilios que presumiblemente se usaban para ese fin. Esta inquietud está presente en todas las culturas y civilizaciones conocidas.

$\mathrm{Si}$ dejamos de lado las revelaciones proféticas y todas aquellas manifestaciones inducidas por drogas o producidas por «estados alterados de conciencia», los métodos utilizados eran de lo más variopinto. Desde consultar las vísceras de animales sacrificados, hasta ver el futuro en la mirada agónica de un prisionero, sacrificado al efecto, que según Estrabón, empleaban los antiguos albaneses y lusitanos.

Los primeros intentos formales de abordar el problema del futuro, surgieron en una etapa precientífica del conocimiento. Tienen en común que parten del principio de analogía en lugar del principio de causa efecto. Suponen un paralelismo entre el mundo «real» y una representación del mismo o «microcosmos» y trasladan los efectos observados del último al primero, como si existiera un sincronismo entre ellos.

Se diferencian entre sí fundamentalmente por el sistema de representación elegido. Entre los más conocidos están los doce signos del zodíaco, los diez sephiroth o las veintidós letras del alfabeto hebreo en la cábala o los veintidós arcanos mayores del tarot, etc.

Científicamente, su valor es puramente antropológico, pero con independencia de sus resultados o implicaciones filosóficas, no se puede hacer una descalificación global de todos ellos. Algunos son auténticas obras maestras del espíritu humano y del pensamiento analógico. Su riqueza simbólica conceptual y su gran fuerza evocadora, son una inagotable fuente de imágenes.

Contrariamente a lo que cabría esperar, este enfoque irracional desde una perspectiva científica y que se puede considerar fruto de la superstición o de residuos de un pensamiento mágico primitivo, propio de las primeras etapas de la humanidad, sigue estando arraigado en amplios sectores de la población. Basta comprobar como los medios de comunicación siguen insertando horóscopos; la razón es que diariamente son consultados por muchos millones de personas.

Lamentablemente, un sistema elaborado durante tantos siglos, no nos sirve para acercarnos al futuro. Sus conclusiones no son demostrables ni repetibles y no han sido constatadas. La ambigüedad de su lenguaje, que le hace aplicable a infinidad de situaciones y de interpretaciones, algunas contradictorias, no le diferencia mucho del de las sibilas de Delfos cuando subidas en el trípode pronunciaban el oráculo. Su carácter hermético, cerrado para los no iniciados, y su lenguaje ambiguo y esotérico, hacen de ellos un campo abonado para charlatanes. 
La prospectiva, una herramienta usada por el Mando

\section{Aportaciones de la ciencia}

La ciencia que es la mejor herramienta que dispone la humanidad para el conocimiento, nace casi incapaz de ayudarnos para estudiar el futuro en el sentido que nos interesa.

Para la física el tiempo es continuo y el principio de uniformidad exige que todos los instantes sean iguales. No existe la concepción del momento absoluto o del instante concreto, el tiempo es duración o intervalo entre dos sucesos. Por otro lado, todas las ecuaciones de la mecánica son invariantes para una transformación de t por - $t$, es decir, aunque el tiempo corriera al revés, se seguirían cumpliendo.

La primera vez que aparece en la ciencia el tiempo con el significado de acontecer es en la termodinámica. El segundo principio aporta un avance fundamental. Un sistema cerrado, el Universo lo es, evoluciona en el sentido en el que aumenta la entropía total del mismo. Esto supone una asimetría y una dirección en el tiempo que señala claramente su sentido, permitiendo distinguir pasado de futuro.

No es casualidad que fuera justamente la termodinámica, la primera ciencia que estudió los sistemas, la que cambiara la situación, permitiendo estudiar la evolución de los mismos. Su enfoque global y sistémico proporcionó avances muy importantes. Por ejemplo en sistemas simples como una reacción química, además de predecir de antemano cual sería el punto de equilibrio, se podía actuar sobre las variables para desplazar este equilibrio dinámico en cualquier sentido hasta alcanzar el punto final deseado, siendo algo tan simple como cambiar adecuadamente la presión y temperatura y controlar las cantidades de reactivos y productos presentes.

Esta doble idea de conocer de antemano y de influir activamente para configurar el futuro deseado, es la base de la prospectiva, como veremos mas adelante. Sin embargo sus métodos son radicalmente distintos: ¿Cuál es el motivo? Si analizamos las condiciones que tenemos en el sistema simple del ejemplo, en primer lugar es un sistema cerrado, y no hay perturbaciones ni acciones externas al mismo que puedan influirle fuera de nuestro control. El sistema se comporta siguiendo unas leyes naturales que existen y que las conocemos perfectamente y por último la ecuación o sistema de ecuaciones que modelan al sistema, tienen solución y podemos obtenerla. Casi ninguna de las condiciones anteriores se cumplen para sistemas complejos. En meteorología por ejemplo, a pesar de que hay modelos muy sofisticados y grandes ordenadores trabajando continuamente, la predicción más allá de una semana pierde toda fiabilidad. La dificultad no es sólo la capacidad 
de cálculo. El problema más que cuantitativo, es cualitativo. Llega un momento en que las soluciones divergen. En sistemas grandes y complejos, es imposible conocer de antemano todas las condiciones iniciales, pero queda una razonable duda de que la naturaleza esté refutando a Laplace y dándole la razón a Poincaré.

Hay una incertidumbre inherente a ciertos sistemas de ecuaciones que hace que muy pequeñas variaciones en las condiciones iniciales, impliquen grandes variaciones en los resultados. Estos sistemas «caóticos», de los que hay muchos ejemplos en la naturaleza, se comportan a efectos globales como si estuvieran regidos por el azar.

Aunque a efectos prácticos no tuvo gran repercusión, el principio de incertidumbre de Heisenberg supuso una conmoción a los pilares mismos de la ciencia. Tardó bastante tiempo en ser aceptado por amplios sectores y no fueron pocas las polémicas que se desataron. Su principal efecto, fue acabar con el determinismo a escala microscópica. No queda claro si la indeterminación es intrínseca a la materia, o sólo se comporta así cuando medimos. Es decir si la indeterminación es causa o efecto. A escala microscópica, no se puede observar sin perturbar lo observado, $\mathrm{y}$ esto hace que lo que se mide venga afectado de un cierto error, imposible de reducir por debajo de cierto nivel. Esta interpretación, pasa de puntillas por el problema del determinismo y se centra en el de la observación. No deja de ser interesante especular con las posibles interpretaciones que puede tener el que el observador necesariamente forme parte del sistema observado, pero esto nos desviaría de nuestro tema. Debido a que el valor de la constante de Plank es muy pequeño, a escala macroscópica no tiene ninguna influencia. Sin embargo, hay algunos sistemas complejos para los que las leyes deterministas no funcionan adecuadamente y no sólo debido a nuestro desconocimiento de las condiciones iniciales ni a las aproximaciones de nuestros cálculos. Parece como si la naturaleza se resistiera a dejarse encerrar en un sistema de ecuaciones. La realidad es compleja y sutil.

Cuando entramos en el campo de las ciencias sociales, la predicción se complica de tal modo que cualquier metodología determinista es inviable. Antes del uso de la prospectiva, se utilizaban fundamentalmente estudios estadísticos de tendencias, con el peligro que supone cualquier extrapolación. La principal dificultad de estos métodos es el hecho contrastado de que el futuro no sólo es evolución, es también mutación y no hay una teoría satisfactoria para el estudio de las mutaciones. Por otro lado estos métodos tienen una limitación de uso exclusivo para predicciones a corto plazo. 
Todo esto, unido a algunos fallos estrepitosos, por ejemplo, la crisis del petróleo de 1973 que cogió a empresas y gobiernos de todo el mundo por sorpresa, hicieron que se empezaran a cuestionar el alcance y utilidad de estos métodos. Los modelos de previsión no son suficientes, hay que complementarlos con el análisis prospectivo que aporta el telón de fondo. Éste cambio de enfoque ha potenciado el desarrollo de los métodos prospectivos.

\section{La prospectiva}

Etimológicamente la palabra prospectiva tiene su origen en el verbo latino «prospiciere» que significa mirar a lo lejos. Surgió a mediados de los años sesenta gracias a Bertrand de Jouvenel. Si a los enfoques anteriores los podemos llamar «fatalista» al primero y «determinista» o «determinista-probabilista» al segundo, el de la prospectiva es «indeterminista». Para ella, el futuro es múltiple e indeterminado y, además, está por hacer. Es decir, no hay un futuro, hay muchos.

$\mathrm{Su}$ visión es global y cualitativa. Su horizonte es a largo plazo e introduce elementos subjetivos en forma de opinión de un conjunto de expertos seleccionados por su conocimiento del tema en estudio. Hay implícita la suposición de que la opinión de un conjunto de expertos, es más válida que la de uno solo. Éste punto, unido a la subjetividad, es todavía fuente de polémica, pero hay que decir por otro lado que no se conoce otra forma satisfactoria de estudiar los saltos o discontinuidades. Lo anterior hace que dentro de La Teoría de Sistemas, se encuadre entre los llamados «sistemas blandos».

La prospectiva supone asimismo un cambio de actitud ante «los futuros», no es suficiente con conocer. El futuro se hace, hay que intervenir. Introduce la figura de los actores, cuyo comportamiento influirá decisivamente.

Sin duda, de todos los futuros posibles hay uno que es el deseable, saberlo con anticipación e intentar poner los medios para llegar a él es el objetivo de la prospectiva. La gran sinergia entre prospectiva y estrategia, hace que se consideren como las dos caras de la misma moneda. La reflexión previa que exige toda toma de decisión, utiliza la prospectiva como uno de sus instrumentos más importantes. Es el anticipar para actuar, pasar de la anticipación prospectiva a la acción estratégica.

Otro punto interesante de la actitud prospectiva, aparte de la reflexión, es el estado de alerta ante los cambios que van apareciendo 
en el horizonte. Es una vigilancia estratégica. Hay que identificar e intentar reconocer los eventos portadores de futuro. Ahí están los embriones del cambio.

\section{Métodos prospectivos}

Los métodos empleados son diversos. Los más conocidos son el método de los escenarios, el método Delphi y los métodos de Impactos Cruzados. Hay además una serie de técnicas auxiliares que ayudan en las distintas fases de los mismos. La elección de uno u otro depende fundamentalmente del tipo de problema que se plantee.

El método de los escenarios consiste en resumen en la elaboración de escenarios pasando por distintas etapas: análisis de sistemas, retrospectiva, estrategia de actores, etc. Se entiende por escenario una situación de futuro y la trayectoria asociada a él. Este método no sólo se ha usado como predicción, ha demostrado gran utilidad en estimular la reflexión colectiva, la motivación y la planificación estratégica de las empresas.

El método Delphi cuyo nombre proviene de la ciudad de Delfos, célebre por sus oráculos, consigue mediante cuestionarios sucesivos, poner de manifiesto convergencias de opiniones y deducir eventuales consensos. Las encuestas se suelen hacer por correo y las respuestas de los expertos son anónimas para garantizar la independencia. Estas respuestas se representan en un histograma y se calculan la mediana y los cuartiles Q1 y Q3. La esencia del método es iterar hasta que se reduce a un determinado nivel el espacio intercuartílico (Q1-Q3). Si esta convergencia no se produce de forma natural, se fuerza mediante técnicas adecuadas.

Los métodos de impactos cruzados, pues hay varios, son los más cuantitativos dentro de los métodos de expertos. En los sistemas reales, donde existen combinaciones múltiples y cambiantes a lo largo del tiempo, de factores humanos, técnicos, sociales, económicos, políticos, etc. interrelacionados, no se puede esperar que los eventos sean independientes entre sí. Estas interrelaciones son las que se tienen en cuenta en forma de impactos cruzados, y de ahí su nombre. El objetivo final es doble, elaborar escenarios y analizar la sensibilidad del sistema de eventos.

En estos métodos hay dos grupos perfectamente diferenciados. Por un lado está el grupo de analistas que es el encargado de elaborar las encuestas y de valorar los resultados y por otro lado el grupo de 
La prospectiva, una herramienta usada por el Mando

expertos que son los que aportan los datos de entrada en forma de probabilidades estimadas de ocurrencia de un conjunto de hipótesis.

Los dos puntos más importantes de todo el proceso son la elección del panel de expertos y la del conjunto de hipótesis. La verosimilitud y pertinencia del resultado final depende del acierto en estas elecciones.

Los expertos se eligen por su reconocida competencia y profundo conocimiento del campo de estudio. Para la elección de las hipótesis se pueden usar técnicas auxiliares como el «brainstorming» o tormenta de ideas; la sinéctica, que ayuda a encontrar ideas totalmente nuevas; el árbol de relevancia, que mediante el análisis sistemático de las relaciones existentes entre los elementos pertenecientes al sistema, y con la ayuda de la teoría de grafos, nos proporciona una representación lógica y jerarquizada de los mismos; la estrategia de actores, que nos permite identificar los objetivos, posicionamiento y táctica de los mismos, y un largo etcétera. Este punto es tan importante que se puede incluso realizar una secuencia delphi para identificar correctamente las hipótesis.

Las hipótesis están bien elegidas, cuando teniendo la máxima independencia entre sí, incluyen a todas las variables clave. Estas hipótesis se pasan a un cuestionario y deben formularse como probabilidad de ocurrencia de un suceso en un horizonte temporal dado. Esta es la encuesta que deben responder los expertos. A efectos de tratamiento matemático del problema, se trabaja con hipótesis y con eventos. Una vez rebasado el horizonte temporal, las hipótesis se convierten en eventos y sólo pueden tener valor uno o cero dependiendo de su cumplimiento o no. Las hipótesis son variables reales, y los eventos variables binarias. De los eventos se sacan los escenarios, y de las hipótesis, las probabilidades de los mismos.

Cada una de las posibles combinaciones de estos eventos, es un escenario. Si hay $n$ eventos, tendremos $2^{\wedge} n$ escenarios. El crecimiento exponencial del número de escenarios impone una limitación en el número de hipótesis. Aunque sólo se analizan los escenarios más probables y los más significativos, hay que cerrar el abanico. No es conveniente que haya muchos para no trabajar con probabilidades muy bajas, pues la suma de las probabilidades de todos los escenarios debe ser igual a uno. Por otro lado si hay pocas hipótesis, los escenarios pueden resultar pobres en su definición. En la práctica se elige una solución de compromiso y se suelen tomar de cinco a nueve hipótesis.

La coherencia de los escenarios obliga a que no haya contradicción entre las probabilidades simples asignadas a las hipótesis y las probabilidades condicionales resultantes de la interacción de las mismas. 
Es decir deben cumplir las leyes de las probabilidades. Esto nunca se cumple en las respuestas dadas por los expertos, para lo cual hay que transformar las probabilidades brutas en otras netas que sí las cumplan y cuyas diferencias con las anteriores sean mínimas. Este paso supone la minimización de una función objetivo cuadrática bajo restricciones lineales y se realiza con la ayuda de programas informáticos.

No sólo interesa estimar la probabilidad de los distintos escenarios, de la información que aportan los expertos, se pueden sacar consecuencias respecto a la importancia relativa de las hipótesis. Mediante matrices de elasticidad, se mide la variación de las probabilidades de los demás eventos, cuando la probabilidad de uno cualquiera varía. Esto se conoce como análisis de sensibilidad y nos permite deducir los eventos dominantes y los dominados, ayudándonos por otro lado a comprender el comportamiento estratégico de los actores, que son los motores del sistema estudiado.

\section{Ejemplo de estudio prospectivo}

Veamos a continuación un ejemplo de aplicación del método de los impactos cruzados. Este ejemplo, tomado de Michel Godet, es muy ilustrativo. Obsérvese como a pesar de las estimaciones falsas del crecimiento económico por parte de los expertos, el estudio sin acertar plenamente, fue de utilidad, presentando por otro lado conclusiones que demostraron su validez. Por supuesto que hay otros estudios prospectivos que vistos a posteriori han resultado más exactos, como el realizado sobre la energía nuclear en el año 2000, pero no es intención de este artículo entrar en valoraciones.

El estudio se realizó en 1974, por encargo del aeropuerto de París y pretendía conocer las tendencias probables del transporte aéreo en la región parisina en el horizonte de 1990. La decisión que estaba en duda era la compra de terrenos para la construcción de un tercer aeropuerto, ante la eventualidad de una respuesta positiva. En este caso, los terrenos deberían de reservarse ya en 1974 e impedir que se urbanizara la zona próxima al mismo.

Las hipótesis que se consideraron fueron, referidas al horizonte de 1990 las seis siguientes:

H1) Más de 50 millones de pasajeros turistas

H2) Promedio de más de 150 pasajeros por vuelo

H3) Retraso medio de despegue superior a 20 minutos 


\section{La prospectiva, una herramienta usada por el Mando}

H4) El precio del billete de avión en valor constante disminuye más del $3 \%$ anual

H5) El PNB francés crece a razón de más del $4 \%$ anual

H6) Limitaciones reglamentarias que provocan una caída del $20 \%$ en el tráfico potencial de vuelos

Estas hipótesis recogían las variables claves del problema, como por ejemplo el incremento del número total de pasajeros turistas. El coeficiente de ocupación de los aviones, y la generalización de aviones de gran capacidad. Que el transporte aéreo se convierta en un transporte de masas, debido a la disminución del precio del billete. La saturación del espacio aéreo. Limitaciones al número de vuelos o prohibición de vuelos nocturnos debido a una mayor sensibilidad o al agravamiento de problemas ambientales.

Las probabilidades absolutas de ocurrencia de las hipótesis que resultaron fueron $0.4 ; 0.7 ; 0.6 ; 0.4 ; 0.6 ;$ y 0.7 respectivamente. Algunas de estas hipótesis han sido desmentidas por los hechos. Por ejemplo se sobrestimaron las perspectivas de crecimiento económico, dando los expertos una probabilidad del 0.6. A pesar de todo, no deja de ser instructivo, y el resultado predijo correctamente cuellos de botella y saturación.

Si numeramos los 64 escenarios resultantes como:

$\mathrm{E} 1(1,1,1,1,1,1)$; (todos los eventos se cumplen).

E2 $(0,1,1,1,1,1)$; (se cumplen todos menos el primero)

E3(1,0,1,1,1,1); (se cumplen todos menos el segundo)

E64(0,0,0,0,0,0); (no se cumple ningún evento)

Los escenarios más probables resultaron:

$\mathrm{E} 1(1,1,1,1,1,1)$ con una probabilidad $\mathrm{P} 1=0.218$

$\mathrm{E} 14(0,1,0,0,1,1)$ con $\mathrm{P} 14=0.110$

$\mathrm{E} 26(0,1,1,0,0,1)$ con $\mathrm{P} 26=0,094$

$\mathrm{E} 12(0,0,1,0,1,1)$ con $\mathrm{P} 12=0.079$

E1 es el escenario en el que todos los eventos se cumplen, es un escenario de conflictos donde hay crecimiento económico y crecimiento del tráfico aéreo.

E14 es un escenario de crecimiento económico con limitaciones reglamentarias que restringen el tráfico. Las compañías generalizan el empleo de aeronaves muy grandes para absorber el crecimiento del tráfico sin aumentar demasiado los vuelos.

Hay escenarios que a pesar de haber obtenido una probabilidad prácticamente nula, son interesantes de comentar. 
$\mathrm{E} 8(0,0,0,1,1,1)$ con $\mathrm{P} 8=0$, al obtener probabilidad cero, indica que no es posible que se den los tres últimos eventos que se consideran variables externas, sin que se de alguno de los tres primeros que son variables internas. Esto significa la sensibilidad del sistema a la evolución ambiental.

E33 $(1,1,1,1,1,0)$ con P33 = 0, es el escenario del crecimiento salvaje y de su probabilidad igual a cero se deduce que los expertos consideran imposible que haya un fuerte crecimiento del tráfico aéreo sin que antes o después se impongan limitaciones reglamentarias.

Del análisis de sensibilidad se concluyó, que el retraso en el despegue y la reglamentación son eventos motores para la evolución del transporte aéreo en la región parisina, siendo la saturación el más sensible. Por otro lado, el precio del billete y el número de pasajeros turistas no tienen un gran impacto, observándose además el uso de aviones grandes no repercute en el descenso del precio de los billetes.

A la vista de los resultados anteriores, cabe preguntarse porqué no se iniciaron los preparativos para construir un tercer aeropuerto si el escenario más probable presagiaba conflictos. Las razones se nos escapan, las decisiones nunca son tan simples, la mayoría de las veces influyen otros factores de conveniencia política, económica o social. No contentos con el resultado que presenta M. Godet en su ejemplo, con los datos aportados y teniendo en cuenta los impactos cruzados, tambien sacados de su ejemplo, los hemos introducido en un programa realizado por nuestro equipo y hemos obtenido otros resultados que se exponen sin ninguna intención de polémica.

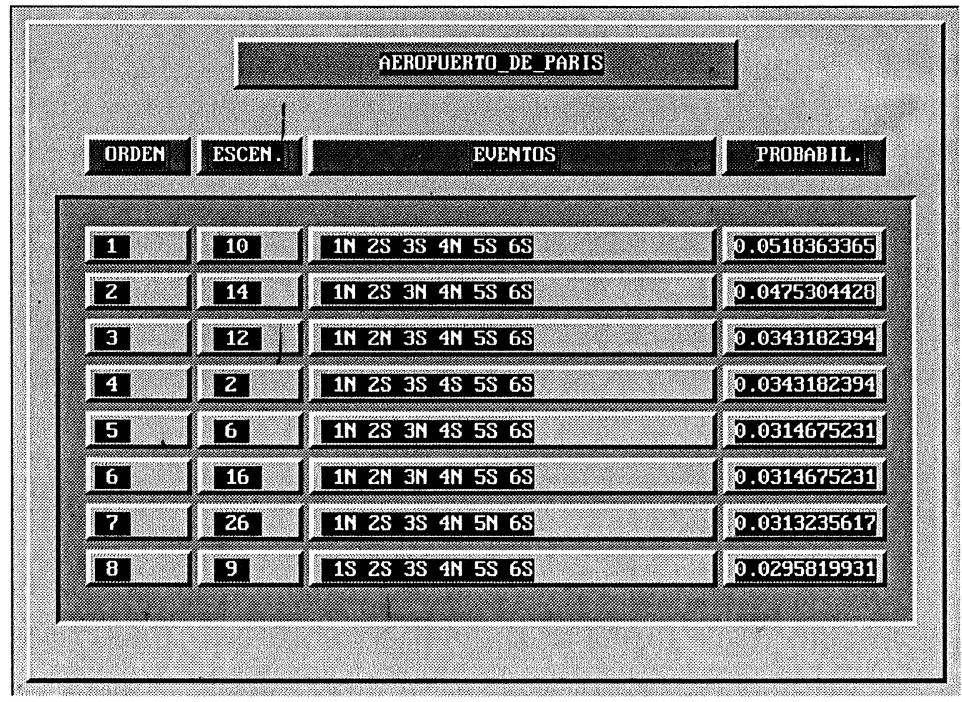


La prospectiva, una herramienta usada por el Mando
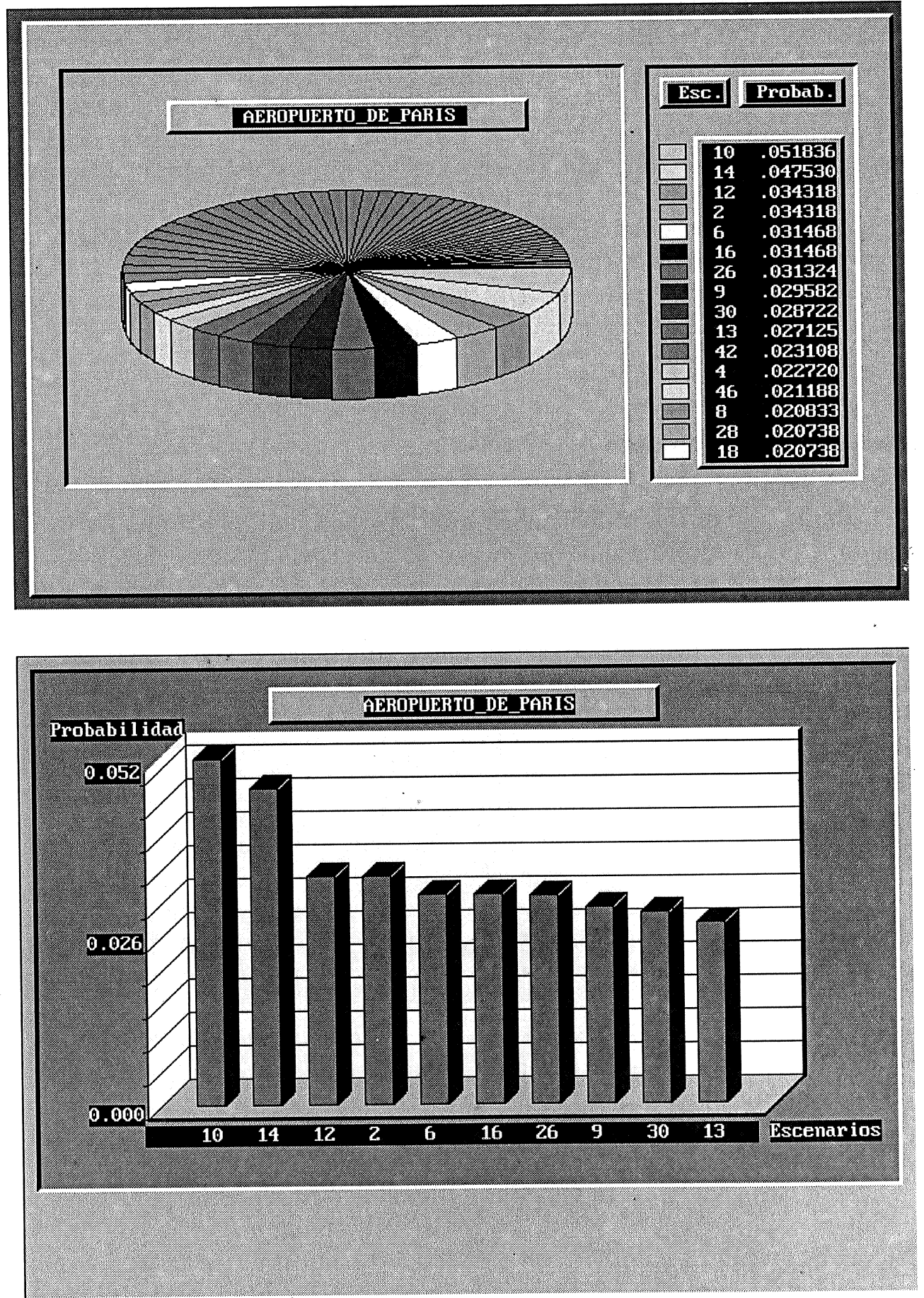
Aquí se ve que el resultado más probable es el escenario $\mathrm{n}^{0} 10$. E10 $(0,1,1,0,1,1)$. Este escenario junto con el E26 son escenarios ecológicos. Hay limitaciones reglamentarias del tráfico que frenan el desarrollo del transporte aéreo, especialmente el turístico. Se generaliza la práctica de llenar los aviones, y hay importantes retrasos en los despegues. En este contexto, no se hace nada para estimular el transporte aéreo y, sobre todo para rebajar el precio del billete.

Un estudio de sensibilidad de las hipótesis da como resultado que los eventos que más influencia tienen sobre la evolución del tráfico aéreo son de mayor a menor $\mathrm{H} 6, \mathrm{H} 1, \mathrm{H} 5, \mathrm{H} 4, \mathrm{H} 2$ y H1. Quizá esto aporte un poco de luz y explique la decisión tomada.

\section{A modo de conclusión}

Cuando se ven las dificultades que suponen cualquier tipo de predicción, cabe preguntarse si realmente es necesaria. ¿Anticipación para qué? ¿Acaso no es suficiente con acomodarse a las modificaciones ambientales imprevistas conforme éstas vayan surgiendo? La respuesta a las anteriores preguntas no es única, depende por un lado de la velocidad con la que se producen los cambios y por otro de la capacidad de reacción. El límite mínimo en la anticipación es el tiempo de respuesta del sistema ante esos cambios ambientales. El trágico hundimiento del Titanic nos puede servir de ejemplo. El iceberg con el que chocó fue visto pero no con la suficiente antelación. Otro barco más pequeño hubiera tenido mas fortuna en las mismas circunstancias. La velocidad y la inercia se conjuntaron con el resultado ya conocido.Las grandes empresas e instituciones tienen una característica de comportamiento, que en un esquema simplista y en primera aproximación, podríamos llamar «inercia». Esta «inercia» es proporcional el tamaño de las mismas, entre otras cosas, y cuando es suficientemente grande, no se puede pasar por alto. A semejanza de las dos primeras leyes de Newton de la mecánica, se puede interpretar como la tendencia a permanecer en el estado de reposo o de movimiento en que se encuentran y como una medida del esfuerzo necesario para realizar cualquier cambio en dicho estado.

Cierta dosis de inercia no sólo es buena, es necesaria para evitar vaivenes peligrosos y modas pasajeras. No olvidemos que cualquier sistema sometido a cambios, está sujeto a respuestas transitorias que pueden ser indeseables o incluso provocar inestabilidad. Por otro lado 


\section{La prospectiva, una herramienta usada por el Mando}

facilita los relevos, y en los «puntos muertos», la inercia permite que siga todo funcionando como si no hubiera pasado nada.

El aspecto negativo de la inercia es el peligro de anquilosamiento, la falta de flexibilidad y las dificultades de adaptación al aumentar el tiempo de respuesta. Este aspecto se hace más patente en épocas de grandes cambios, como es la actual donde la evolución de la situación internacional, la aparición de nuevos conceptos estratégicos y el rápido avance de la tecnología hace que métodos y medios queden obsoletos en muy poco tiempo. Sólo hay dos maneras prácticas de reducir la inercia, disminución de tamaño y anticipación.

Reducir el tamaño, aumentando simultáneamente la capacidad operativa, no es contradicción, es cambiar cantidad por calidad y se consigue simplificando la organización y mejorando la formación, los medios y los procedimientos.

La anticipación requiere un proceso de reflexión y análisis con profundidad y amplitud, además de una vigilancia de los factores de cambio. Esto se consigue con la prospectiva.

Un paso más dentro de la dinámica de adaptación a entornos inciertos y cambiantes con el tiempo, es imitar la capacidad que para ello tienen los seres vivos, y desarrollar sistemas que en alguna medida se comporten análogamente. La principal característica en este sentido es la capacidad de aprendizaje, es decir utilizar las experiencias adquiridas en el pasado, incluso los errores cometidos, para modificar las actuaciones y decisiones en el presente.

Cuando distintos órganos, inicialmente dispersos, se coordinan y unifican esfuerzos, agilizando a su vez la relación entre ellos, se consigue lo que en teoría de sistemas se llama sinergia. El todo resulta mayor que la suma de las partes.

Vemos, pues, desde la óptica de la teoría de sistemas, como el Ejército del siglo XXI, con exigencias cada vez más elevadas respecto a eficiencia, operatividad, precisión en sus actuaciones y amplitud en el rango de sus misiones, se ha reorganizado, y se ha dotado de un órgano integrante del apoyo a la Fuerza, el MADOC, que le permitirá cumplir su misión en condiciones óptimas, con independencia de las modificaciones ambientales. Del MADOC dependen directamente, la Dirección de Investigación y Análisis para el Combate; la Dirección de Doctrina, Orgánica y Materiales y la Dirección de Enseñanza, Instrucción, Adiestramiento y Evaluación.

La Dirección de Investigación y Análisis del MADOC, tiene un equipo de analistas dedicado a estudiar las "lecciones aprendidas», y otro equipo ha iniciado varios estudios prospectivos que están en marcha 
en la actualidad. Cuando éstos terminen, se dispondrá de unos escenarios de futuro que servirán de base de reflexión y al reducir las incertidumbres, ayudarán en la toma de decisiones.

\section{Bibliografía}

Ayres, R. U., «Technological Forecasting and Long-Range Planning», New York: McGrawHill, 1969.

Berger, G., «L'attitude prospective», París, Puf, 1967.

Dewar, J. A., y Levin, M. H. «Assumption-Based Planning for Army 21», Santa Monica, Calif.: RAND, R-4172-A, 1992.

Dewar, J. A., Builder, C.H., Hix, W. M. y Levin, M. H. «Assumption-Based Planning», Santa Monica, Calif.: RAND.

Godet, M., "De la anticipación a la acción. Manual de prospectiva y estrategia», Barcelona: Marcombo, 1993.

Instituto Español de Estudios Estratégicos, «Investigación de fenómenos belígenos: Método analítico factorial», Cuadernos de Estrategia N. ${ }^{\circ}$ 17, MD, 1990.

Instituto Español de Estudios Estratégicos, "Análisis factorial de las causas que originan los conflictos bélicos", Cuadernos de Estrategia N. 69, MD, 1994.

Katsuhiko, O., "Ingeniería de control moderna", Prentice-Hall Hispanoamericana S. A. México, 1980.

Martino, J.P., «Technological Forecasting for Decisionmaking», New York: Elsevier, 1972. Toffler, Alvin, «The Adaptativ Corporation», New York: McGraw-Hill,1985.

TorRón Durán, R., «La Prospectiva y su metodología». Memorial Ingeniería de Armamento $\mathrm{n}^{\circ}$ 77. 1980.

Torrón Durán, R. «El Análisis de Sistemas». Publicaciones de Ingeniería de Sistemas n. ${ }^{\circ} 16$. Isdefe. 1997. 